\title{
Experiencias intensivas de trabajo de campo como herramienta pedagógica para la conservación patrimonial en ciudades marginalizadas
}

Intensive Fieldwork Experiences as a Pedagogical Tool for Heritage Conservation in Marginalized Cities

Experiências intensivas de trabalho de campo como ferramenta pedagógica para a conservação patrimonial em cidades marginalizadas

Edwar Calderón*

Dimitris Theodossopoulos **

Recibido: 29 de abril del 2020

Aprobado: 24 de noviembre del 2020

https://doi.org/10.12804/revistas.urosario.edu.co/territorios/a.9002

Para citar este artículo:

Calderón, E., \& Theodossopoulos, D. (2021). Experiencias intensivas de trabajo de campo como herramienta pedagógica para la conservación patrimonial en ciudades marginalizadas. Territorios, (44 Especial), 125-154. https://doi.org/10.12804/revistas.urosario.edu.co/territorios/a.9002

\begin{abstract}
* Universidad EAFIT, Colombia. Correo electrónico:eacalderoc@eafit. edu.co. ORCID: https:// orcid.org/0000-00027881-8131

* Escuela de Arquitectura y Paisajismo (ESALA), Universidad de Edimburgo, Escocia. Correo electrónico: d.theodossopoulos@ed.ac. uk. ORCID: https://orcid. org/0000-0002-91796950
\end{abstract}


Palabras clave

Conservación arquitectónica; trabajo de campo; patrimonio históricocultural; herramientas pedagógicas inclusivas; educación en ciudades marginalizadas; Quibdó-Colombia.

Keywords Architectural conservation; fieldwork; historicalcultural heritage; inclusive pedagogical tools; education in marginalized cities; Quibdó-Colombia.

Palavras-chave Conservação arquitetônica; trabalho de campo; patrimônio histórico-cultural; ferramentas pedagógicas inclusivas; educação em cidades marginalizadas; Quibdó-Colômbia.

\section{territarias 44 Especial}

\section{RESUMEN}

El trabajo de campo en la educación para la conservación del patrimonio arquitectónico se considera principalmente una herramienta para documentar la condición actual y desarrollar habilidades relevantes como metodología comprometida con los valores arquitectónicos, culturales e históricos de un sitio. Sin embargo, su pedagogía, en ausencia de un amplio debate, puede enriquecerse mediante la revisión de experiencias en Geografía y Arquitectura, donde el trabajo de campo, una actividad esencial en los planes de estudio de pregrado, induce a los estudiantes a realizar preguntas críticas a través del aprendizaje y mejora la participación con lo inesperado a través de la interrupción de los procesos de formación lineales. Esta metodología ha demostrado producir experiencias de enseñanza intensivas en conservación patrimonial a través de talleres, por lo que este trabajo reflexiona sobre cómo pueden mejorarse estas pedagogías y cómo estas pueden aportar significativamente a la sostenibilidad del patrimonio cultural en poblaciones que han sido históricamente marginalizadas de los principales centros urbanos.

\section{ABSTRACT}

Fieldwork in architectural conservation education is mainly considered a tool to document the current condition and develop relevant skills, as a methodology committed to the architectural, cultural and historical values of a site. However, their pedagogy, in the absence of a broad debate, can be enriched by reviewing experiences in Geography and Architecture, where fieldwork, an essential activity in their undergraduate study plans, induces students to ask critical questions through learning and enhance engagement with the unexpected by disrupting linear learning processes. This methodology has been shown to produce intensive teaching experiences in heritage conservation through workshops, so this work reflects on how these pedagogies can be improved and how they can significantly contribute to the sustainability of cultural heritage in cities that have been historically marginalized from the main urban centers.

\section{RESUMO}

O trabalho de campo na educação para a conservação do patrimônio arquitetônico é considerado principalmente uma ferramenta para documentar o estado atual e desenvolver competências relevantes, como uma metodologia comprometida com os valores arquitetônicos, culturais e históricos de um local. No entanto, sua pedagogia, na ausência de um amplo debate, pode ser enriquecida com a revisão de experiências em Geografia e Arquitetura, onde o trabalho de campo, uma atividade essencial em seus planos de estudo de graduação, induz os alunos a fazerem perguntas críticas por meio do aprendizado e aumenta o engajamento com o inesperado por meio da interrupção dos processos de formação lineares. Esta metodologia demonstrou produzir experiências pedagógicas intensivas em conservação patrimonial por meio de oficinas, portanto, este trabalho reflete sobre como essas pedagogias podem ser aprimoradas e como podem contribuir significativamente para a sustentabilidade do patrimônio cultural em cidades historicamente marginalizadas dos principais centros urbanos. 


\section{Introducción}

Este manuscrito reflexiona sobre el valor pedagógico que pueden tener los ejercicios de trabajo de campo cuando son cortos e intensivos en la enseñanza (o la introducción) de la disciplina de la conservación arquitectónica en los estudiantes de educación superior en zonas marginalizadas, donde el patrimonio histórico frecuentemente no adquiere las dimensiones necesarias para su sostenibilidad cultural, a la vez que discute rutas de mejoramiento de esta metodología de aprendizaje. El detonante de esta reflexión fue un taller de conservación organizado para una escuela joven de arquitectura en Colombia, por un grupo de académicos de las escuelas de Arquitectura (ESALA) y Geociencias de la Universidad de Edimburgo, con el apoyo financiero del Concejo Británico, Reino Unido. La experiencia del trabajo de campo fue vista como una actividad pragmática y efectiva por la aplicación del aprendizaje que tuvieron los estudiantes, a pesar del corto periodo de enseñanza, y para los autores, las actividades demostraron ser exitosas tanto durante escuelas de verano como en cursos de posgrado. El taller en Colombia tuvo por objetivo generar capacidad de enseñanza sostenible en conservación arquitectónica como eje primordial en el cuidado y preservación del patrimonio arquitectónico local para su plan de estudios de pregrado, por lo cual, este manuscrito argumenta atributos para un mejoramiento del programa de arquitectura.

El trabajo de campo en educación para la conservación es un método típico para desarrollar habilidades como levantamiento arquitectónico, una actividad intensiva y breve para recopilar datos geométricos y de patología de un sitio, pero también el vehículo más directo para iniciar una fase metodológica que se compromete con la arquitectura existente (Brandi, 2005; Carbonara, 2012; Matero, 2007) generando curiosidad por la naturaleza de lo construido. Sin embargo, puede desarrollarse aún más en la investigación crítica a través del "aprender haciendo" y luego pasar a un proceso de formación completamente nuevo, "trabajar" con lo existente, abierto a la incertidumbre y la interrupción, inclusive puede hacer que los estudiantes cambien sus prácticas de observación, análisis y síntesis (Consejo Internacional de Monumentos y Sitios, ICOMOS, 1993). Esto se evidencia en las prácticas de los programas de Arquitectura y Geografía que enseñan cómo ver a las personas existentes, estudiar sus culturas y su vínculo con patrones y cambios en el entorno natural para promover el aprendizaje activo y el desarrollo de los estudiantes como investigadores (France \& Haigh, 2018). Adicionalmente, este método puede producir hallazgos inesperados que alimentan aún más la curiosidad y la investigación crítica.

La conservación es vista como una disciplina especializada que surgió de la territarios 44 Especial 127 
${ }^{1}$ University of Edinburgh. Land Use Planning Challenges in the Informal City: the Case of Quibdo's City Centre 2019. School of Geosciences. http:// www.geos.ed.ac.uk/geosciences/research/projects/ land-use-planning-challenges-informal-citycase-quibd\%C3\%B3s-citycentre

${ }^{2}$ Universidad Tecnológica del Chocó (UTCh, 2019). Pensum, Programa de Arquitectura. https:// www.utch.edu.co/portal/ es/pensum-programa-arquitectura.html

\section{territarias 44 Especial} 128 práctica profesional, generalmente enseñada en Europa a nivel de posgrado, aunque cada vez con más auge en los planes de estudio de pregrado de arquitectura como cursos o electivas, siguiendo la tradición histórica en países como Italia o el desarrollo de la integración europea en la educación superior (Musso \& De Marco, 2007). En un contexto de posgrados como el escocés, significa un mayor grado de complejidad y profundidad en el aprendizaje y, los primeros pasos en la autonomía y abstracción de la investigación de un alumno (QAA Scotland, 2012). Dado que se involucran muchas habilidades profesionales y artesanales, la conservación debe enseñarse con un enfoque holístico como actividad interdisciplinaria que mejora en última instancia la conciencia cultural y la sensibilidad de los estudiantes (ICOMOS, 1993). No obstante, se espera que la mayoría de los alumnos de programas de arquitecura ejecuten la mitad de sus proyectos en edificios existentes. Los estudiantes de posgrado en conservación vienen de una variedad creciente de campos académicos, como lo demuestra la amplia admisión de la Maestría en Conservación Arquitectónica en ESALA.

Los ejercicios de trabajo de campo cortos e intensivos han demostrado ser vehículos de aprendizaje muy fuertes y efectivos desde la experiencia de los autores, que en general, incluyen desde el estudio de un edificio y su patología, hasta proponer una intervención que varía entre la reparación y el diseño. Dentro de un curso de conservación, esto puede producir una interacción crítica directa con los valores de los entornos arquitectónicos existentes, en cualquier grado de la historicidad de un sitio (Venturi et al., 1972), mientras que en un curso de diseño puede ser un estímulo creativo generado a partir de pistas como la descomposición, resiliencia, estratificación, destrucción, testimonio de interacción humana, memoria, etcétera (Carbonara, 2011). No todos los programas comparten una pedagogía especial del trabajo de campo, ya que consideran solo la función básica de este último como la recolección de datos y la evaluación del sitio para el desarrollo del curso.

Una oportunidad para reflexionar sobre el trabajo de campo surgió después de una colaboración internacional entre la Universidad de Edimburgo y la Universidad Tecnológica del Chocó (UTCh), con el fin de establecer un taller de pregrado sobre la preservación de edificios históricos en Quibdó, el cual mostró un compromiso muy fuerte de los estudiantes. Este taller hizo parte de un proyecto más amplio para comprender las prácticas locales y las relaciones intersectoriales mediadas por el cambio en el uso del suelo en la ciudad de Quibdó en Colombia, ${ }^{1}$ y se organizó para mejorar la capacidad de enseñanza en conservación del programa de pregrado de Arquitectura en la UTCh. ${ }^{2}$ Para este propósito, se condensaron la estructura y la evaluación del curso mencionado de 
la Universidad de Edimburgo, ${ }^{3}$ (encuesta, resumen histórico, patología, propuesta de análisis y reparación). El enfoque del taller fue sobre un patrimonio descuidado y fragmentado, pero altamente significativo y sensible en Quibdó, casas hechas en madera de finales del siglo XIX (Cujar, 2015; González, 2003; González, 2004).

El esfuerzo por adaptar el curso a un taller de cuatro días reunió diversas culturas académicas. El fuerte compromiso de los estudiantes mostró el entusiasmo por el tema y, los tutores que lideraron los equipos tenían las habilidades profesionales necesarias. Los testimonios de la resultante creciente conciencia de su patrimonio, el interés por hacer que el patrimonio reviva, y el protagonismo que adquirió el centro histórico tradicional de la ciudad como laboratorio para sus futuros proyectos, es un indicador del éxito del taller. Lo anterior, junto con las experiencias previas en la enseñanza a nivel de posgrado en Edimburgo o en escuelas de verano en diferentes localidades, alentó a los autores a reflexionar en este documento respecto a cómo tales experiencias intensivas de aprendizaje de trabajo de campo son exitosas y cómo se puede mejorar su práctica actual en educación para la conservación, pero aún más, reflexionar acerca de los resultados de esta metodología en un contexto tan diferente del europeo, en este caso el Chocó colombiano y, cómo esta puede contribuir a prácticas académicas más incluyentes en pro de la sostenibilidad cultural en ciudades marginalizadas, como lo ha sido Quibdó históricamente. Finalmente, la preparación del taller fue tan importante como la entrega, ambos demostraron la importancia de conocer el sitio, los sistemas y materiales de construcción, pero también la cultura académica local, la de una escuela de arquitectura pequeña y joven en una región relativamente excluida.

Muchos educadores están de acuerdo en que los especialistas en restauración necesitan desarrollar una capacidad crítica, más allá de la evaluación de las características técnicas o los datos ambientales de un sitio (Jokilehto, 2007). De una manera similar sucede con el aprendizaje del diseño arquitectónico, donde se producen relaciones estrechas de los estudiantes con el entorno del sitio, pero están aún enmarcados desde un concepto de proyecto. Es decir, estimular el pensamiento crítico puede producir una interrupción de la transmisión lineal de conocimiento cuando está genuinamente abierto a la incertidumbre, lo que hace que el aprendizaje sea contextual y más rico, y lleva a una propiedad y confianza más temprana en el manejo de lo inesperado, que es una habilidad particularmente útil en la disciplina de la conservación. Estos son aspectos pedagógicos que el trabajo de campo puede mejorar.

Así mismo, el taller demostró que una preparación básica de pregrado en la tecnología arquitectónica contemporánea puede ayudar a que las habilidades de conservación especializadas se absorban de manera
${ }^{3}$ University of Edinburgh. MSc in Architectural Conservation. Degree Programme Table. http:// www.drps.ed.ac.uk/18-19/ dpt/ptmscarconlf.htm 
efectiva, por ejemplo, entender mejor el proceso de fabricación de componentes en la construcción. La calidad de lectura de los edificios estudiados cambió; la mayoría de estudiantes produjo un análisis sorpresivamente exhaustivo, y este aspecto durante el levantamiento de la información, mostró que las ideas preconcebidas culturales o técnicas se pueden dejar de lado durante tales actividades. Por lo tanto, un objetivo adicional en este manuscrito es reflexionar sobre cómo esta metodología puede ser una experiencia refrescante dentro de un plan de estudios de Arquitectura, que promueva compromisos más duraderos con el patrimonio arquitectónico.

\section{Experiencias de aprendizaje en trabajos de campo de duración breve y alta intensidad productiva}

Los trabajos de campo en conservación introducen a los estudiantes a comprender el patrimonio construido dentro de su realidad local. Aquí el diseño arquitectónico se puede enmarcar como un "contexto" que extrae, posiciona y articula estas relaciones críticas en un proyecto (Ewing, 2012). El trabajo de campo es esencial para los cursos donde los estudiantes aprenden ciertas técnicas (levantamiento, análisis de edificios) o recopilan datos para un estudio (diseño urbano, tecnología de la construcción). Algunos programas de posgrado ofrecen largos viajes de campo al extranjero en los cuales se exploran prácticas de conservación en otros lugares o talleres especializados intensivos, tales como excavaciones arqueológicas y campañas de restauración o construcción tradicional. La multidisciplinariedad en este campo se enseña en algunos casos a través de proyectos de diseño grupal con estudiantes de diferentes disciplinas profesionales, y eventualmente, involucran actividades intensas de trabajo de campo (Fiorani, 2019).

La enseñanza a través del trabajo de campo generalmente está dirigida por la información existente en muchos programas de conservación $\mathrm{y}$, se ha producido poca reflexión en la literatura - como los foros educativos de la Asociación Europea para la Educación en Astronomía (EAAE) - (Musso \& De Marco, 2007). Es por esto que la consideración de este manuscrito sobre la ampliación de esta experiencia de aprendizaje, es explorada desde la pedagogía de la educación arquitectónica y geográfica, en lugar de la arqueológica donde, de manera similar a la conservación, se basa en el aprendizaje de habilidades prácticas.

Las salidas de campo en los planes de estudio de Arquitectura tienen como objetivo mapear e interpretar la información de un sitio, e incluso, pasar de la documentación objetiva a varios métodos para interactuar con su carácter. En ESALA, por ejemplo, se espera que los estudiantes en su segundo año mapeen los aspectos más esenciales de las áreas que estudian en Valencia en un deambular casi situacionista (Bonet, 2019); en el tercer año, 
se les pide que recopilen sus propios set de datos digitales en todo el casco antiguo de Edimburgo para desarrollar técnicas de diseño que reconozcan la multiplicidad de agencias y subjetividades individuales que sustentan la vida moderna de la ciudad (Paredes \& Bonet, 2018). En la Maestría de Arquitectura (MArch-Part Two), en un módulo de proyectos, se crearon en primer lugar modelos ambientales en las condiciones controladas de un taller de la escuela para su estudio, que se convirtieron en instrumentos posteriores para diseñar edificios cuando las respuestas simuladas se contextualizaron en el entorno real y extremo de Islandia (Moffit, 2019). Tales enfoques para "ver", "conocer" y eventualmente "trabajar" con las prácticas existentes mediante el trabajo de campo, tienen el potencial de transformar el observador al "romper" hábitos, habilidades y crear otros nuevos (Ewing, 2012), similares a la geografía como se verá más adelante.

Posteriormente, diseñar intervenciones en conservación puede hacer ver "lo existente" como algo más que una "edificación existente", al tener en cuenta la experiencia de la esencia de un lugar y reconocer las técnicas históricas y los hábitos de trabajo, así se pueden crear nuevos contextos particulares que fortalecen el vínculo del edificio histórico con la vida moderna.

La geografía humana puede considerarse como una disciplina cercana a la conservación, que se beneficia enormemente de "aprender haciendo" en escenarios reales, por lo tanto, el trabajo de campo juega un papel central en sus programas académicos. De esta manera, el trabajo de campo se ha establecido como una actividad obligatoria para la educación en Geografía en Inglaterra y Gales, desde la década de 1990, para lo cual Bland et al. aseguran que "la geografía sin trabajo de campo es como la ciencia sin experimentos" (1996, p. 165). Es una poderosa herramienta pedagógica que permite a los estudiantes confrontar perspectivas teóricas con entornos de la vida real y su importancia está en constante evolución en los programas de estudios (figuras 1 y 2 ), e informa también de otras disciplinas, como se ha discutido ampliamente en la literatura de educación superior (Gold, 1991; Smith, 1987; Foskett, 1997; Foskett, 1999; Fuller et al., 2006; France \& Haigh, 2018; Kent et al., 1997).

La revisión de los debates contemporáneos acerca de la importancia del trabajo de campo en los procesos de aprendizaje, proporciona algunas observaciones relevantes para el desarrollo futuro de esta pedagogía en la conservación (Dunphy \& Spellman, 2009; France \& Haigh, 2018; Fuller et al., 2006; Scott et al., 2006). En corcordancia, Fuller et al. (2006) examinaron cuatro estudios de caso: dos en el Reino Unido (Chester y Liverpool), uno en Singapur y, otro en Nueva Zelanda. En todos se llegó a la conclusión de la efectividad del trabajo de campo en los programas de estudio de geografía. En Nueva Zelanda, por ejemplo, tersitorios 44 Especial 131 
Figura 1. Cambios en los enfoques del trabajo de campo en Geografía 1950-1997

Date Approach

1950 Traditional 'look-see' or 'Cook's tour field, courses

- observational and descriptive

1960 - 'landscape'-based or centred on 'sight-seeing' visits to specific sites of interest in geography

- passive student participation

1970 'New' Geography-1960s 'revolution'

Problem-orientated, project-based fieldwork

- inductive and deductive approaches (positivist) hypothesis generation and testing, data collection and statistical analysis, interpretation and report writing

1980 - detailed scales, often carried out in a small area

- active student participation although often staff-led

1985 Enterprise in Higher Education-Transferable skills

Problem-orientated fieldwork still dominant but Introduction of transferable skills element

- project design skills

- organisational skills

- leadership skills

- group skills

- active student participation but emphasis switches from staff-led to student-led projects

1990 Massive growth in student numbers-teaching large classes

- Field courses incorporate elements of all previous modes of fieldwork

- may commence with look-see' perhaps combined with thematic guided walks/trails

- followed by staff-directed, problem-orientated projects

- then student-initiated problem-centred work with added dimension of transferable skills

1997 Serious problems of cost of fieldwork to both Departments and students combined with even larger classes

- the future?

- 'virtual reality' to assist with field courses

- but will 'virtual reality' be any cheaper or ever be as satisfactory?

Fuente: Kent et al. (1997). 
Figura 2. Siete modos principales de trabajo de campo en Geografía de educación superior

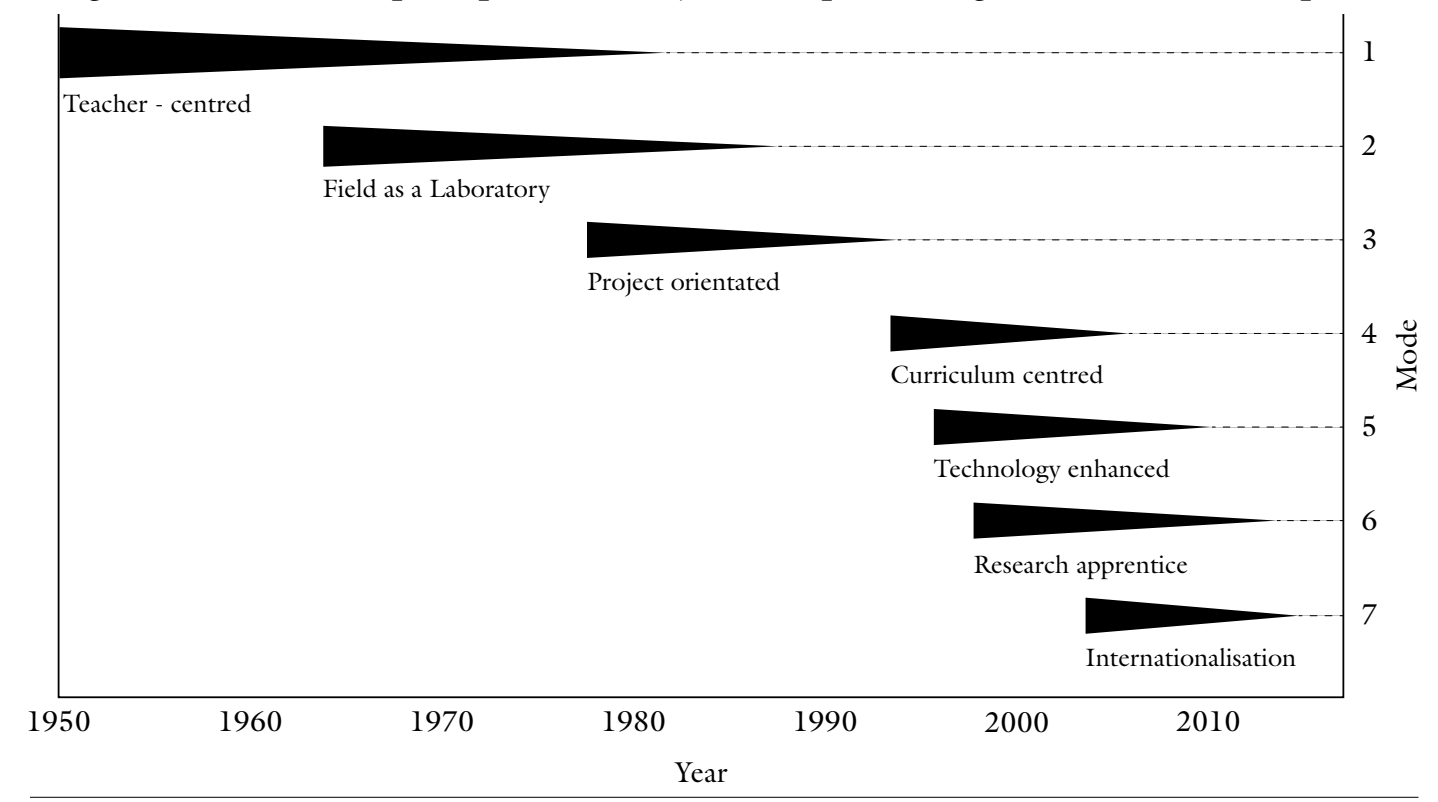

Fuente: France y Haigh (2018).

cuestionaron la competencia del trabajo de campo en la década de 1990 (cuando se percibía como un método de aprendizaje pasivo en lugar de activo) y promovieron una reorientación de este enfoque, como la asignación de ejercicios de solución de problemas en grupos pequeños en lugar de toda la clase; un cambio similar ocurrió en otras regiones como el Reino Unido. Un estudio previo de Kent et al. (1997) y más recientemente, France y Haigh (2018), apoyaron este argumento (véase figura 1 y 2). Su revisión crítica sobre la metodología del trabajo de campo en las universidades del Reino Unido desde la década de 1950, pone especial énfasis en la participación directa tanto de docentes como de estudiantes. Ambos estudios señalaron el paso de los estudiantes de dependientes a autónomos como uno de los resultados más importantes de este cambio.

Con respecto al desarrollo de los estudiantes, Smith (1987) observó el valor del trabajo de campo como un método de enseñanza en tres categorías: "indagaciones al aire libre", "estudios al aire libre" y "progreso personal y social". En particular, la primera categoría puede proporcionar a los estudiantes una conciencia de su entorno a través de la experiencia física fuera del aula. Los "estudios al aire libre" promueven la aplicación y adquisición de nuevos conocimientos en tersitorias 44 Especial 


\section{territorias} 44 Especial un contexto específico que estimula el crecimiento intelectual. Además, como señaló Foskett (1997), también estimula "el desarrollo de geografías personales a menudo identificadas como el desarrollo de un 'sentido de lugar', que a su vez exige la experiencia y la participación en el 'lugar', tanto en entornos familiares como desconocidos" del alumno (p. 191). Por último, el "progreso personal y social" se trata de la responsabilidad social y la capacidad de trabajar en colaboración (Foskett, 1997; Smith, 1987) o como argumentan Fuller et al. (2006), quienes ven el trabajo de campo como una herramienta de aproximación cercana entre estudiantes y profesores para el estudio de un tema.

Por otro lado, un análisis DOFA dentro del trabajo de campo como un enfoque pedagógico en geografía humana (Hope, 2009) muestra que es un 'modo valioso de aprendizaje', pero con un aspecto 'poco teorizado': su valor a veces está respaldado por experiencias circunstanciales particulares en lugar de ser 'objetivo y basado en la investigación’ (Nairn, 2005; Foskett, 1999).

\section{Transferencia de conocimiento a través del diálogo posgrado-pregrado}

El taller de conservación en Quibdó fue visto como una experiencia enriquecedora que requiere reflexión sobre la práctica del trabajo de campo. Metodológicamente, por motivos prágmaticos de tiempo y costos, se condensó un curso de posgrado en una semana de intensa actividad. Así se discutieron las formas en que la educación de posgrado en la conservación, puede alimentar los currículos de diseño en pregrado y, en particular, cómo pueden apoyar al desarrollo académico en el programa de pregrado de la UTCh.

La mayoría de los programas de conservación en Europa son a nivel de posgrado, y en general, se basan en ciudades con un gran patrimonio arquitectónico, lo que hace que el trabajo de campo sea gratificante. Así mismo, recopilan la experiencia de investigación de los tutores en un plan de estudios especializado, lo que va más allá de los ofrecimientos curriculares de los programas de pregrado tradicionales y la necesaria interacción con los organismos de acreditación profesional. Un título de maestría también proporciona experiencias de aprendizaje a cohortes de estudiantes muy diversos, pero enfocados en una escala de tiempo desafiante, experiencias caracterizadas por una creciente complejidad e independencia en la investigación, el aprendizaje y oportunidades para hacer abstracciones más sensatas (QAA Scotland, 2012). Los posgrados se dividen en currículos con capacitación integral (por ejemplo, ESALA Edimburgo, Sapienza Roma, ETSA Madrid, Lovaina y Bamberg), y aquellos que se basan en prácticas contemporáneas de diseño (Bartlett) o historiografía (ETH Zurich).

La maestría en ESALA es un programa que se enseña sin un enfoque específico 
de diseño, pero contiene historia y teoría, tecnología, levantamiento y análisis, conservación urbana, y culmina con una disertación. Ahora bien, la asignatura central "Tecnología de la conservación", en particular, es dirigida por el segundo autor que aborda los principales materiales tradicionales y modernos, y practica aspectos como mantenimiento, sostenibilidad y regulaciones. Se evalúa mediante un informe técnico de estudios de caso estructurado a lo largo de levantamientos arquitectónicos, evaluación del valor cultural, análisis de patologías y propuesta de reparación. Como no todos los estudiantes tienen formación técnica, el curso cubre la patología de materiales y reparaciones a nivel cualitativo.

Con respecto a la enseñanza a nivel pregrado, en países de patrimonio arquitectónico altamente rico como Italia, las escuelas de arquitectura incluyen clases de conservación en el plan de estudios, como la segunda parte del programa Laurea Magistrale en Architettura (Restauro) en Roma Sapienza. ${ }^{5}$ Algunas universidades prefieren concentrarse en aspectos como la ciencia y la tecnología (muchos programas en Alemania o cursos en Politecnico di Milano). Un aspecto implícito de la educación para la conservación es que los estudiantes interactúan en una escala $1: 1$ con un edificio existente, lejos de contextos irreales de diseño donde se crean nuevos edificios. Además, los entornos históricos con riqueza arquitectónica como Roma o Edimburgo ofrecen indicaciones complejas para proyectos de diseño de pregrado a cualquier nivel, como decadencia, resiliencia, fragmentación, estratificación, destrucción, testimonio de interacción humana, memoria, identidad fragmentada, entre otras (Carbonara, 2011), como lo muestra el currículo de Maestría en Arquitectura (MArch) en ESALA. Esto muestra la necesidad de enmarcar la conservación con mayor precisión en la enseñanza en pregrado, como un proceso que los arquitectos practicarán en su carrera profesional, de manera similar al debate sobre disciplinas como el diseño urbano, la arquitectura del paisaje, la fabricación digital, entre otras, ya sea con asignaturas electivas o diferentes alternativas de profundización.

El trabajo de campo es otra actividad que conecta la conservación con los planes de estudio de diseño en cursos de pregrado, y es fundamental para el espíritu de la Maestría de Edimburgo, la cual involucra a los estudiantes en el registro de geometrías y patologías, visitando sitios en construcción, lugares arqueológicos o explorando desarrollos urbanos. Por ejemplo, para la asignatura "Análisis de edificios", se espera que los estudiantes "demuestren conocimiento en la variedad de métodos de documentación y competencia para aplicarlos al levantamiento y el análisis de edificios históricos", mientras que para "Tecnología de la conservación", los estudiantes deberían poder "mostrar habilidades en la elaboración de levantamientos de las condiciones de una construcción". ${ }^{6}$ Esto
${ }^{4}$ University of Edinburgh. (2019). Conservation Technology: Degree regulations and programmes of study (DRPS). http://www. drps.ed.ac.uk/19-20/dpt/ cxareal1017.htm

${ }^{5}$ Universitá di Roma "La Sapienza”. (2019). Laurea Magistrale in Architettura (Restauro). https:// corsidilaurea.uniromal. it/it/corso/2019/29846/ bome

territarias 44 Especial

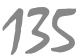


${ }^{6}$ University of Edinburgh. MSc in Architectural Conservation. Degree Programme Table. bttp:// www.drps.ed.ac.uk/18-19/ $d p t / p t m s c a r c o n l f . h t m$

${ }^{7}$ University of Edinburgh. Land Use Planning Challenges in the Informal City: the Case of Quibdo's City Centre 2019. School of Geosciences. http:// www.geos.ed.ac.uk/geosciences/research/projects/ land-use-planning-challenges-informal-citycase-quibd\%C3\%B3s-citycentre

\section{territarias 44 Especial}

se enmarca en el contexto más amplio de la práctica educativa recomendada por ICOMOS (1993): “La conservación requiere la capacidad de observar, analizar y sintetizar" (pp. 4-5) o el resumen de los atributos de un profesional de la conservación definido por el Institute of Historic Building Conservation (IHBC): "Investigación, registro y análisis".

Cuando se estableció el proyecto $D e$ safíos en la planificación del uso del suelo en Quibdó, liderado por el primer autor en el 2016, ${ }^{7}$ uno de los objetivos era evaluar los procesos y prácticas en construcción de vivienda, y cómo estas se adaptaban a las condiciones sociales y climáticas de la ciudad de Quibdó, - el campo de investigación de la arquitecta y docente Johana Lozano-Maya, quien coordinó esta actividad (Lozano, 2019) - . Después del primer taller sobre los impactos socioeconómicos y ambientales del cambio en el uso del suelo en el centro tradicional de Quibdó y su sostenibilidad, la conservación se consideró como el siguiente paso para comprender la dinámica histórica y mejorar las prácticas locales de sostenibilidad. Este taller enriquecería el plan de estudios de la escuela de arquitectura de la UTCh y fortalecería el compromiso de los estudiantes con su contexto local, con el objetivo de convertirse más adelante en una plataforma para la colaboración creativa con las organizaciones de la sociedad civil y las autoridades locales como una aproximación incluyente y sostenible.
Las huellas de la arquitectura de madera de finales del siglo XIX fueron identificadas como testigos de los procesos que crearon y transformaron a la ciudad, su tejido social y construcción. Algunas viviendas palafíticas se construyeron sobre cañadas que luego serían altamente contaminadas, por lo que sus áreas se convirtieron en umbrales y barrios marginales que necesitan regeneración (Lozano, 2019). Los tutores de la UTCh indicaron que los estudiantes de último año serían los alumnos apropiados para esta actividad. El taller permitiría, por lo tanto, que los estudiantes se involucraran con su herencia, que la escuela explorara metodologías y que su capacidad de enseñanza e investigación mejorasen en esa dirección. Para los autores fue una oportunidad de probar aspectos del aprendizaje de conservación a nivel de pregrado y evaluar los intercambios culturales que tienen lugar cuando las pedagogías establecidas se trasladan a un entorno muy diferente (EdimburgoQuibdó), incluso desde una perspectiva descolonial, es decir, desvinculando una aproximación al conocimiento impuesto desde Europa. El trabajo de campo fue visto como una actividad estimulante, así mismo, fueron reconocidas características técnicas y culturales significativas del uso histórico de la madera en la construcción de edificios en la región que serán discutidas en otra publicación (Arango, 1993; Cujar, 2015; González, 2003, 2004).

El programa de Arquitectura en la UTCh se estableció en 1997, por lo que es 
relativamente joven y puede innovarse con las tendencias actuales. Su plan de estudios (pensum), es definido como una "estructura integradora de disciplinas", una composición de resultados de aprendizaje, habilidades profesionales y transferibles, estrategias de enseñanza y métodos de evaluación. Además, el programa apunta a una estrategia de enseñanza flexible que responde a las necesidades de la comunidad y la promoción del desarrollo humanístico y académico de los estudiantes, para abordar las necesidades arquitectónicas que surgen a nivel local y nacional. ${ }^{8} \mathrm{El}$ programa se divide en cuatro grupos de asignaturas - diseño, tecnología, historia y teoría, práctica profesional-, bastante típico de acuerdo con las tendencias internacionales del plan de estudios profesional y similar a ESALA; además, se complementa con asignaturas electivas —urbanismo y medio ambiente, vivienda social, tecnología avanzada, sostenibilidad- que representan el $25 \%$ del total de créditos.

En los años superiores, el programa se traslada a proyectos de investigación de diseño dirigidos por los intereses y la experiencia de los tutores, como "Arquitectura y Hábitat", "Arquitectura bioclimática” y "Urbanismo y desarrollo territorial". Sin embargo, la respuesta a las "condiciones y particularidades locales" que promueve la filosofía del programa es muy variable. Una unidad de diseño, por ejemplo, se centra en edificios de viviendas de gran altura que responden muy poco a cualquier contexto local y a los muchos problemas de la ciudad, tales como la precariedad en sus servicios básicos de saneamiento y agua potable. Otros proyectos se desarrollan en sitios alejados de la ciudad, probablemente sin ver el potencial del diseño arquitectónico en las áreas urbanas actuales. Esto cuestiona qué tan significativos los tutores ven el impacto de la innovación arquitectónica en el desarrollo de la ciudad.

En resumen, el programa de enseñanza está fuertemente orientado hacia el desarrollo profesional y técnico y, parece responder menos a las condiciones climáticas y socioeconómicas locales, la preservación histórica o los materiales de construcción naturales. El programa no parece ofrecer a los estudiantes suficientes oportunidades para desarrollar habilidades de levantamiento, no cuenta con la infraestructura necesaria, no tiene buena conexión a Internet en el campus, ni una sección con recursos suficientes sobre arquitectura en la biblioteca o talleres básicos de maquetas. Promover proyectos utópicos en el aula de clase puede significar para algunos estudiantes dificultades para leer el contexto local y proponer soluciones arquitectónicas que respondan a una necesidad imperiosa de soluciones sostenibles; en cambio, algunas de las materias esenciales que abordan estas necesidades solo están disponibles como clases opcionales para ellos.

En un contexto más amplio, la marginalidad educacional de la región ha hecho que la escuela carezca de programas de posgrado o de investigación, lo que no
${ }^{8}$ Universidad Tecnológica del Chocó (UTCh, 2019). Pensum, Programa de Arquitectura. https:// www.utch.edu.co/portal/ es/pensum-programa-arquitectura.html territarios 44 Especial 137 
${ }^{9}$ University of Edinburgh. Land Use Planning Challenges in the Informal City: the Case of Quibdo's City Centre 2019. School of Geosciences. http:// www.geos.ed.ac.uk/geosciences/research/projects/ land-use-planning-challenges-informal-citycase-quibd\%C3\%B3s-citycentre

\section{territorias 44 Especial} 138 estimula la realización de investigación independiente de la enseñanza. Tampoco se financian proyectos relacionados con la madera, arquitectura bioclimática o hábitat, lo que hace que la escuela parezca esencialmente un departamento de enseñanza vocacional.

\section{Trabajo de campo en arquitectura de madera: taller de conservación en Quibdó}

Elegir el tipo de trabajo de campo más apropiado fue fundamental para el proceso de aprendizaje. La revisión anterior de literatura sobre trabajos de campo en Geografía en educación superior (France \& Haigh, 2018; Kent et al., 1997), mostró que una combinación de diferentes tipos de esta actividad podría ser más efectiva en algunos contextos geográficos. Siguiendo con el objetivo de nutrir las habilidades y la conciencia patrimonial en el taller de Quibdó, el trabajo de campo fue útil no solo para los estudiantes de pregrado sino también para sus educadores. Así pues, con el propósito de dejar la capacidad de la enseñanza de la asignatura instalada, se consideró apropiado un trabajo de campo de observación participante guiado por docentes, es decir, un punto medio entre un proyecto tradicionalmente liderado por docentes y un trabajo grupal independiente (figura 1). Lo anterior puede incorporar estas actividades más explícitamente en un currículo basado en la investigación o en la solución de un problema (Pawson et al.,
2006) y promover modelos de aprendizaje conjunto (Le Heron et al., 2006; Fuller et al., 2006).

Si bien algunos de estos aspectos se hicieron evidentes durante el taller, la etapa de planificación apuntó a un ejercicio que podría mejorar la sensibilidad y las habilidades del alumno para leer su entorno histórico y atraerlo a través de la interacción directa (Smith, 1987). El taller dividió a los estudiantes en grupos de 10 personas, cada grupo recibió dos edificios para inspeccionar, analizar y documentar, luego harían una propuesta de renovación o conservación, cuando fuera necesario. La mayoría de estos edificios de madera están aislados en un entorno construido de finales del siglo $\mathrm{xx}$, por lo que esta experiencia puede conducir al desarrollo de geografías nuevas, personales y basadas en el diseño, más allá de emular las pocas publicaciones académicas al respecto (Cujar, 2015; González, 2003, 2004). También se planificó como una oportunidad para implementar el conocimiento impartido en conferencias cortas en el primer día, siguiendo inicialmente un patrón de aprendizaje lineal.

Otros resultados de aprendizaje en línea con el proyecto de investigación más amplio, ${ }^{9}$ fueron el desarrollo de la responsabilidad social de los estudiantes a través de una mayor conciencia del patrimonio y su papel en el carácter urbano de la ciudad para su sostenibilidad. La reflexión sobre las técnicas y los hábitos pasados para crear edificios que se basan más en la artesanía, 
puede influir en sus propios métodos de diseño y ampliar el contexto en el que generalmente funcionarían.

Actualmente la mayoría de los edificios de la ciudad son de hormigón y ladrillo tras la modernización y reconstrucción, después de un gran incendio en 1966 (como se puede observar en la figura 3 ). Esto ha ido eliminando gradualmente la madera en los edificios, que caracterizó a la ciudad desde su mayor desarrollo a fines del siglo XIX. La ciudad está ubicada a lo largo del río navegable Atrato, el cual creó las condiciones adecuadas para el comercio y los vínculos culturales con Panamá y el Caribe, por lo que se convirtió en la capital de la región del Chocó desde 1770, un área inmersa en la selva del Pacífico, abundante en madera y minerales.

Desde 1876 muchas casas urbanas se construyeron en madera en una tipología importada en gran parte del Caribe, junto con madera aserrada de la zona y zinc (Cujar, 2015; González, 2004). Esto produjo edificios de 2 pisos más complejos, con técnicas de construcción avanzadas (sistemas de entramado, cerchas de techo, placas de pared, marcos de apertura) o características modernas y expresivas como aleros y balcones en madera o metal. Para este ejercicio, se seleccionaron 6 de aproximadamente 15

Figura 3. Panorámica de Quibdó desde el norte

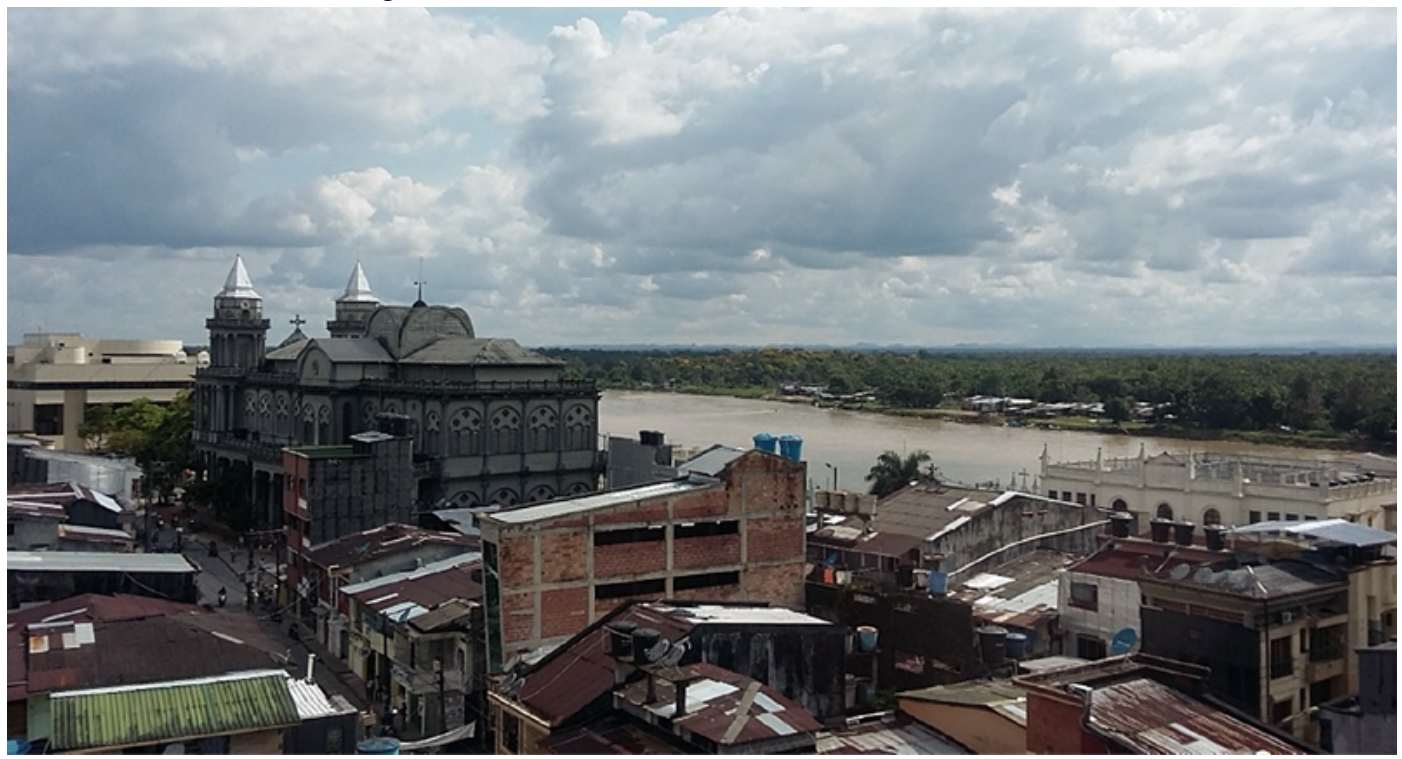

Nota: se muestran casas típicas y los principales edificios monumentales (Catedral San Francisco de Asis, Banco de la República y Diócesis).

Fuente: elaboración propia. 
casas de madera históricas preservadas (figura 4), de acuerdo a las fechas de construcción, condición y accesibilidad para los estudiantes.

El taller tuvo una duración de cinco días (del 24 al 28 de abril del 2017) con estudiantes en 3 grupos diferentes dirigidos por tutores seleccionados. La preparación esencial se realizó en Edimburgo, con el uso de textos clave del patrimonio histórico de Quibdó (Cujar, 2015; González, $2003,2004)$ y visitas previas del primer autor. El servicio Street View ${ }^{\circledR}$ de Google Maps fue una herramienta fundamental para la preparación del material de la conferencia, la familiarización con los tipos de edificios y la escala urbana de la ciudad. Se envió previamente un informe resumiendo las tareas para los estudiantes y tutores, con el apoyo de lecturas sobre técnicas históricas (Huerta, s.f.) y patología de la madera (Lasheras, 1998).

\section{El taller}

El primer día inició con la presentación del programa del taller y la divisón de los 30 estudiantes en tres grupos, cada uno liderado por un tutor/docente. Luego se dictaron tres charlas estilo conferencia: la primera, impartida por el segundo autor, trataba sobre preservación arquitectónica y patología de la madera; la segunda, por Douglas Cujar-Castañedas, arquitecto experto en arquitectura e historia urbana de la zona, proporcionó una visión tersitorias 44 Especial
Figura 4. Trabajo de campo en Quibdó

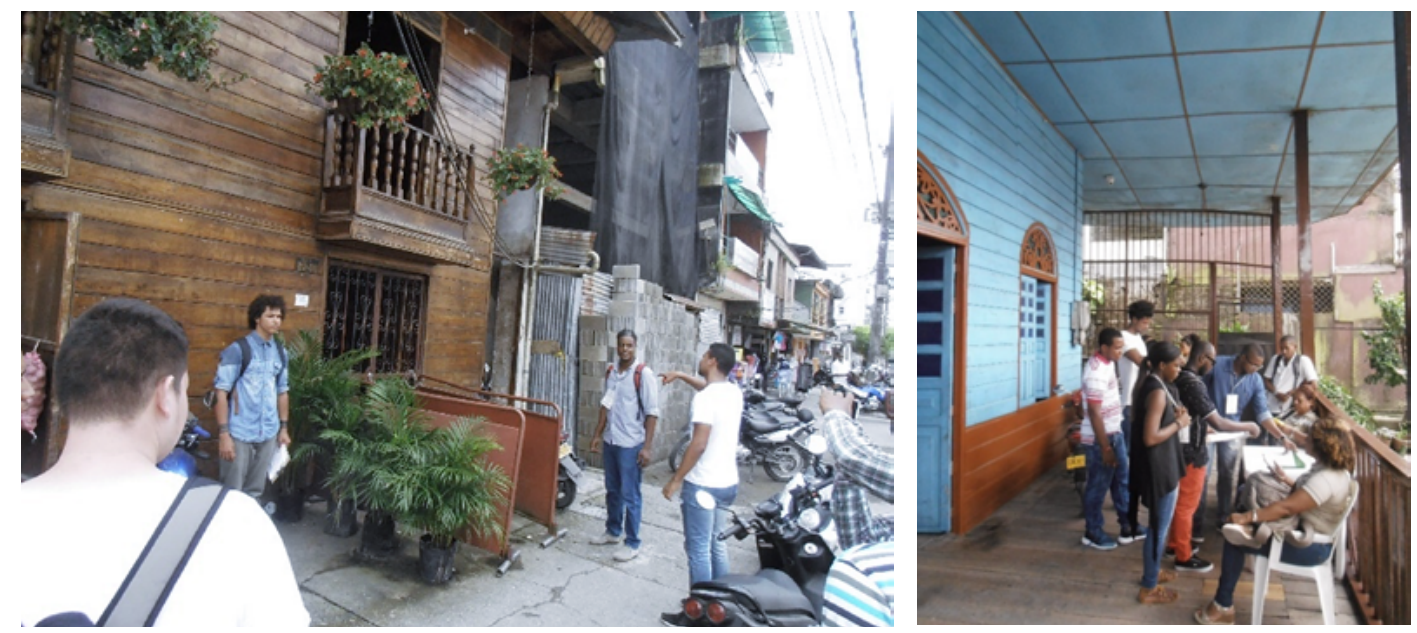

Nota: estudiantes de la UTCh durante el levantamiento arquitectónico de los patrones de composición típicos. Izquierda: la muy bien conservada Casa Copete (Carrera 5 y Calle 27, barrio Cesar Conto). Derecha: Casa Medina (Carrera 7 y Calle 24, barrio La Yescagrande). 
significativa de los edificios históricos y el desarrollo urbano de Quibdó (figura 5), y la tercera, por la arquitecta Johana Lozano, directora del programa de arquitectura en la UTCh, presentó su investigación sobre tipologías de viviendas en zonas inundables y no inundables en Chocó, ampliada por sus alumnos.

El segundo día comenzó con una visita al principal hito histórico de la ciudad, la catedral San Francisco de Asis, construida entre 1946 y 1979, y la Casa de la Diócesis construida entre 1931 y 1942 (véase figura 6), guiados por el padre Napoleón García, quien vinculó el desarrollo de la comunidad afrodescendiente en Quibdó con la historia de la catedral y sus diferentes etapas de construcción. Luego, los estudiantes pasaron el resto del día inspeccionando sus edificios asignados (figura 4).
El análisis comenzó el día tres. En la mañana, el equipo de Edimburgo se reunió con estudiantes y tutores para organizar y planear el proceso de levantamiento de información. En términos generales, los estudiantes habían logrado inspeccionar bien sus edificios el día anterior, lo que les dio más tiempo de procesar la información para la presentación final, y así, trabajar en la escuela con sus tutores.

El análisis posterior continuó el cuarto día, los estudiantes comenzaron a preparar sus presentaciones para la revisión. En la tarde, los grupos presentaron sus análisis y propuestas en el auditorio con la ayuda de pósters (figura 7). Los propietarios de las casas estudiadas fueron invitados a la revisión, en especial, la propietaria de Casa Medina, quien generó una discusión muy significativa sobre la importancia de preservar los edificios de madera en

Figura 5. Charla-conferencia durante el primer día del taller
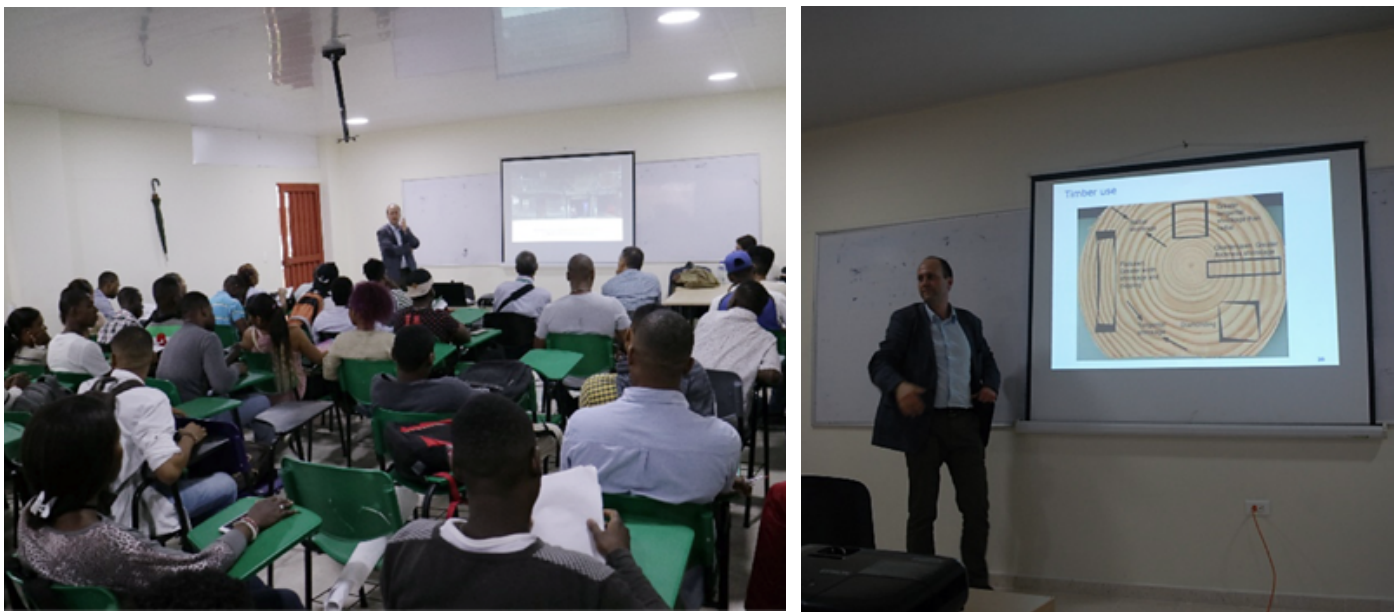

Fuente: elaboración propia. 
tersitorias

44 Especial

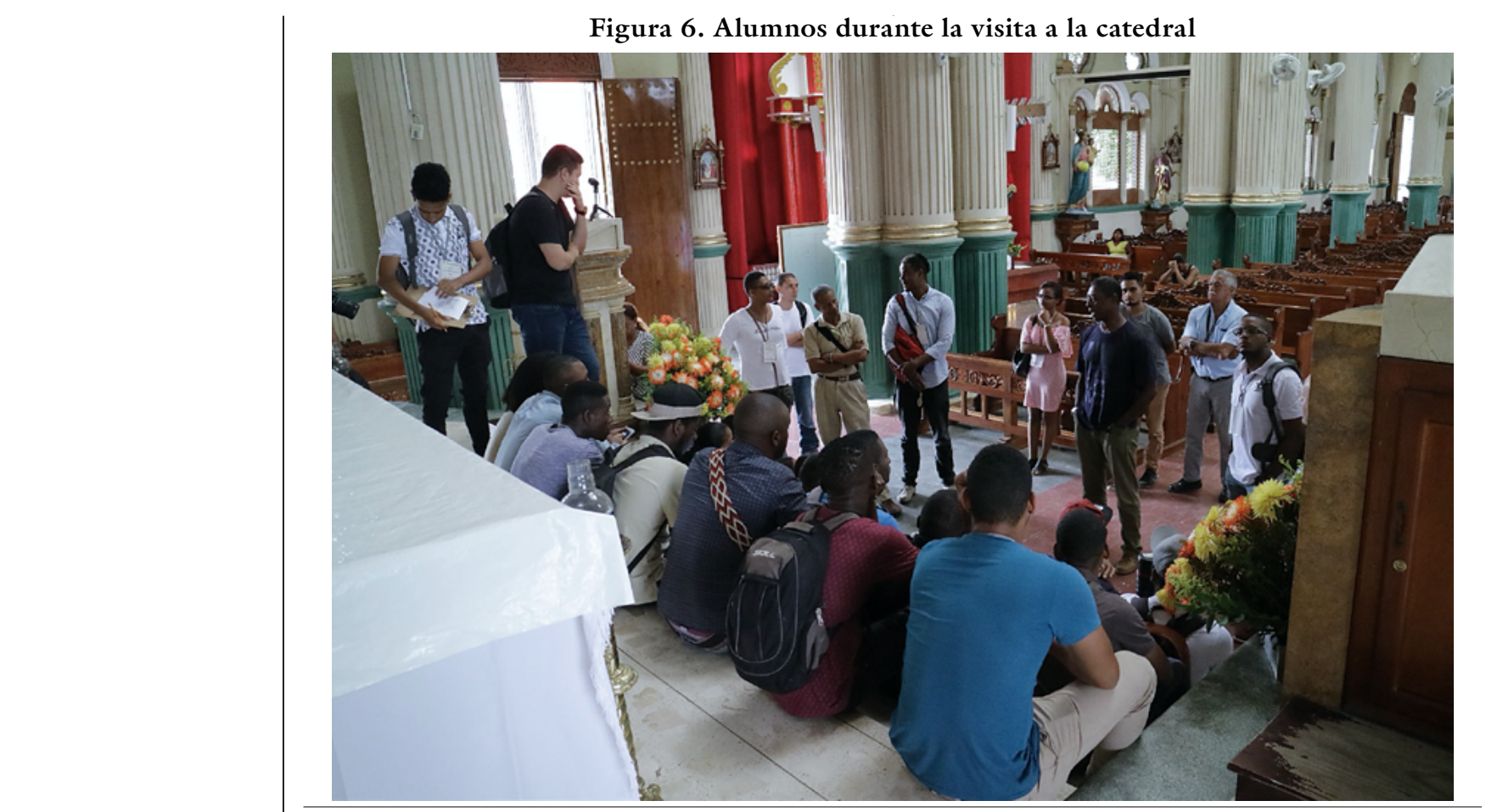

Fuente: elaboración propia.

Figura 7. Ejemplos del trabajo de los estudiantes y la exhibición final

Figura 6. Alumnos durante la visita a la catedral
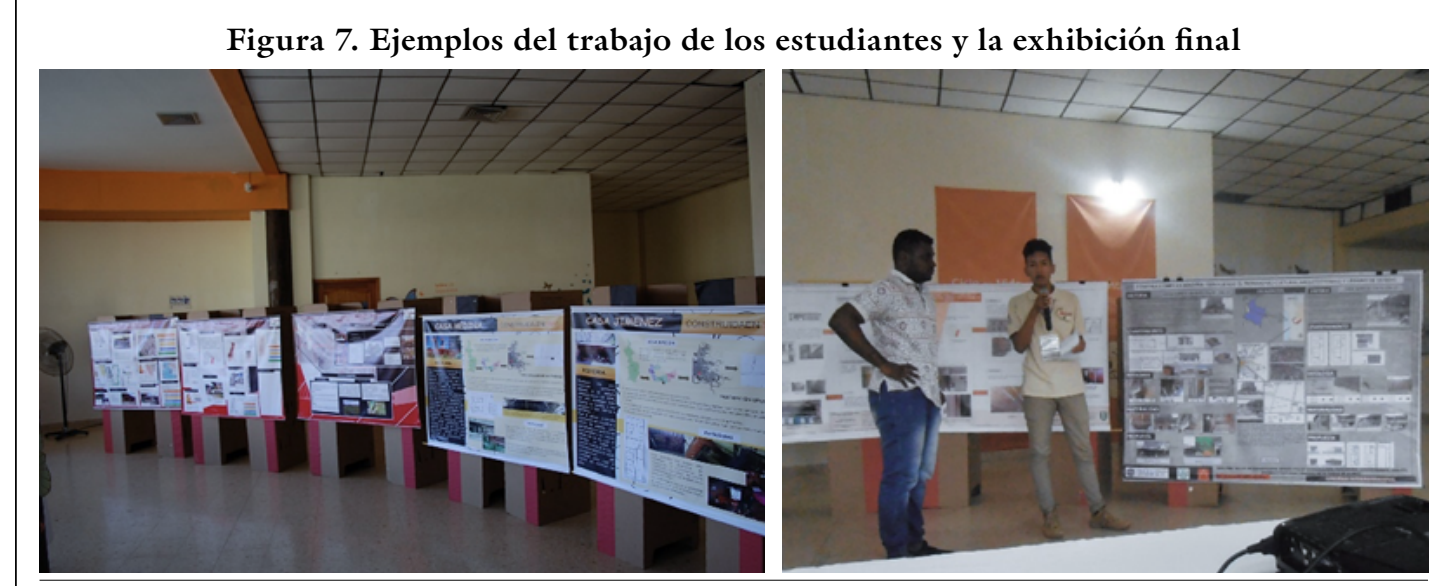

Fuente: elaboración propia. 
Quibdó. La respuesta de los estudiantes al ejercicio fue muy atractiva y las revisiones mostraron vínculos claros entre la calidad del levantamiento arquitectónico y la profundidad del análisis.

Los pósters se dejaron exhibidos para ser presentados el día siguiente (día 5) a las autoridades locales -Oficina de planificación de la ciudad, CODECHOCO y Gobernacion del Chocó- y a los dueños de los aserraderos de madera. La exhibición terminaría en una discusión abierta con los invitados. Sin embargo, no hubo presencia de ellos, lo que llevó a que la dicusión propuesta fuera principalmente académica.

\section{Beneficios académicos de una corta e intensiva experiencia de aprendizaje en Quibdó}

El taller en sí puede considerarse un éxito. Esta breve actividad estimuló un amplia gama de participación de estudiantes y tutores, produjo aprendizaje y habilidades en conservación, y estimuló observaciones interesantes en edificios específicos, lo que aumentó la conciencia del patrimonio entre los estudiantes. El análisis de Casa Copete y Casa García fue extenso (figuras 4 y 7 ), impulsado no solo por el levantamiento arquitectónico y el estudio de su historia, sino también por consideraciones de diseño como la materialidad, lo que condujo naturalmente a enriquecedoras propuestas de conservación.
La ausencia de las autoridades durante el debate público limitó nuestra visión de propiciar una oportunidad de debate y discusión más amplia, que pudiera conllevar a un mejoramiento en los procedimientos cívicos (política pública) en torno al patromonio histórico de la ciudad. No obstante, se logró crear conciencia sobre cómo la conservación puede mejorar el enfoque holístico del plan de estudios arquitectónico. Se discutieron varios puntos sobre la conciencia de este patrimonio maderero entre los propietarios, en específico (Casa Medina) o en general (conciencia del rendimiento técnico y la importancia cultural de los materiales regionales como Guayacán para postes o, palma (Chonda) para techos impermeables). Se planteó la falta de mano de obra en la construcción maderera y la necesidad de resaltar más la cultura de los materiales vernaculares (regionales) en las asignaturas de tecnología. Hacer que el patrimonio viva y tenga significancia se convirtió en una declaración muy fuerte por parte de algunos estudiantes, quienes expresaron la necesidad de utilizar el centro de la ciudad como laboratorio para sus estudios. Así mismo, por parte de todos los participantes se manifestó que la investigación debería centrarse en la historia de la ciudad y la región, actualizada con el estudio de las nuevas características que se crean en la periferia de la ciudad, donde la construcción de casas sobre palafitos se convierte en la práctica actual para las territarios 44 Especial 143 
comunidades víctimas del desplazamiento forzado, o las relaciones de los nuevos barrios construidos para estas comunidades con el centro de la ciudad.

Este patrimonio de la construcción en madera está bastante fragmentado en toda la ciudad, no obstante, durante una visita previa del primer autor, pudo reconocer al menos 15 propiedades de valor arquitectónico - aunque es probable que hayan más-. Aún así, las técnicas de tableros de madera utilizadas se pueden encontrar en edificios y cabañas modernas de menor tamaño. Identificarlos por su mérito arquitectónico particular, incluso como huellas de la arquitectura urbana antes del incendio de 1966, puede eventualmente permitir que los estudiantes los vean como una capa importante que está oculta por la arquitectura de hormigón no regulada que siguió desde entonces (figura 8). En ocasiones, esto puede enriquecer las relaciones críticas en sus proyectos de diseño al crear una conciencia renovada del contexto urbano, como se espera de las actividades de trabajo de campo.

Enmarcar el taller en el debate contemporáneo (figura 2) sobre la importancia del trabajo de campo en los procesos de aprendizaje (France \& Haigh, 2018; Dunphy \& Spellman, 2009; Fuller et al., 2006; Scott et al., 2006), muestra algunos avances adicionales. La asignación de pequeños ejercicios de solución de

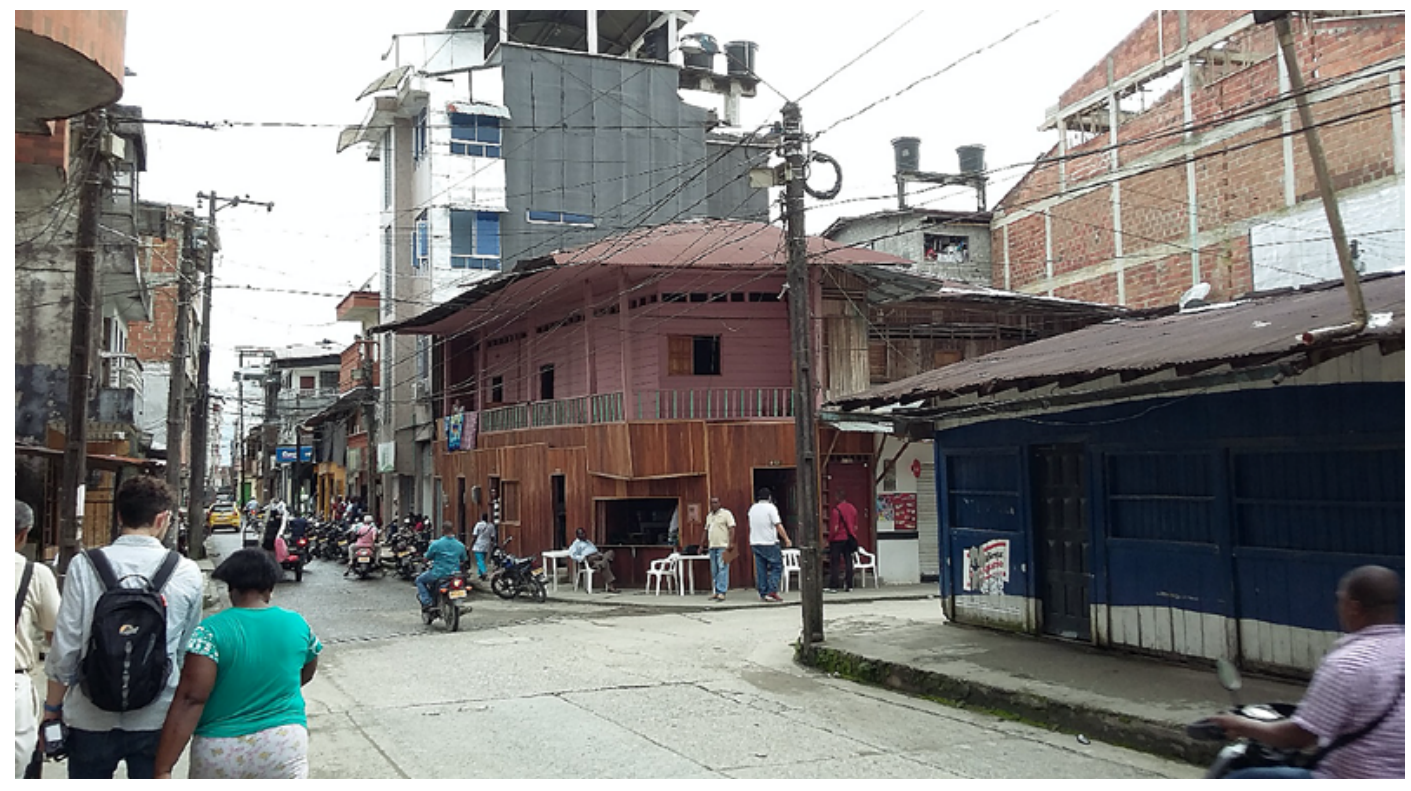

Fuente: elaboración propia. 
problemas en grupos más pequeños en lugar de toda la clase (Fuller et al., 2006) promovió una participación más activa, mejorada aún más por el aporte directo del tutor (France \& Haigh, 2018). Las presentaciones y la discusión al final del taller confirmó el progreso de los estudiantes al demostrar que gradualmente se volvieron más seguros y autónomos. Se produjeron muchas ideas independientes que evidenciaron cómo la metodología de trabajo de campo elegida estimulaba su autonomía en el pensamiento crítico y el desarrollo de nuevas habilidades - es decir, levantamiento de edificios e identificación de patología de materiales-.

La importancia de utilizar un enfoque basado en la solución de problemas reales, se demuestra de manera clara por los resultados logrados en Quibdó, en especial, en la participación activa de los estudiantes y sus tutores durante y después del trabajo de campo. El equipo de Edimburgo apoyó a los estudiantes con la selección de estudios de caso y material didáctico que podría aplicarse directamente en su conservación. A pesar de ello, los estudiantes eran libres de analizar cualquier aspecto relevante o priorizarlos en consecuencia durante el trabajo de campo - es decir, identificación y mitigación de patologías de construcción-.

Los resultados iniciales del trabajo de campo en Quibdó son prometedores, pero aún se deben evaluar los impactos a mediano y largo plazo. Dichos resultados brindan un fuerte apoyo a la evaluación de
Smith (1987) respecto al valor del trabajo de campo como método de enseñanza en tres categorías: indagaciones al aire libre, estudios al aire libre y desarrollo personal y social. Los anteriores están intrínsecamente conectados y si se implementan en el plan de estudios de la UTCh, producirían habilidades transferibles a través del desarrollo personal y social de los estudiantes que repercutirían en futuros profesionales interesados por la sostenbilidad sociocultural y medioambiental - por el uso de materiales vernaculares renovables - de la ciudad a través de la conservación patrimonial.

Ahora bien, con respecto al potencial para el desarrollo de capacidades en la UTCh, todavía tiene que materializarse. La buena preparación de los estudiantes mostró que el plan de estudios de pregrado de la escuela ofrece suficientes componentes que pueden transformarse en cursos especializados. Pero el compromiso crítico irregular con el contexto de la ciudad, así como la falta de un círculo de especialistas en conservación o referencias en la biblioteca - que cuenta con pocos recursos-, son condiciones limitantes. Sumado a la falta de programas de maestría o doctorado y la investigación no muy extensa en la escuela, en áreas relevantes (madera, arquitectura bioclimática, hábitat urbano). Incluso, si la UTCh quiere aparecer como una universidad de enseñanza vocacional que promueve enfoques prácticos, la formación de arquitectos necesita tutores con curiosidad y espíritu crítico, y esta es territarios 44 Especial 145 
un área adicional que el trabajo de campo puede mejorar.

La discusión sobre las acciones político-administrativas que dicho patrimonio necesita hoy, resaltó la necesidad imperativa de una normativa estricta en Quibdó: un listado del patrimonio y su cumplimiento, actualización y enfoque del Plan de Ordenamiento Territorial local (РОT) y promoción de rutas turísticas. El interés generado durante el taller sobre estos edificios domésticos, ciertamente se suscribe a cambios similares de énfasis en toda Colombia en los últimos 30 años (Arango, 1993). Desde entonces se han adoptado enfoques críticos para las modificaciones históricas del patrimonio, creando experiencias arquitectónicas en el diseño de la conservación. Estos aspectos fueron explorados por los estudiantes e instintivamente generaron ideas para hacer que dichos edificios sean relevantes para su práctica de diseño, y expandir el canon de la conservación al patrimonio moderno asociado con las preocupaciones contemporáneas, como la inclusión y la sostenibilidad. Entonces, ¿podría el taller crear una "conciencia histórica" en el enfoque de los estudiantes sobre el entorno urbano de Quibdó? Indudablemente.

Figura 9. Palafitos en la Quebrada La Yesca, barrio La Yescagrande

tersitorias 44 Especial
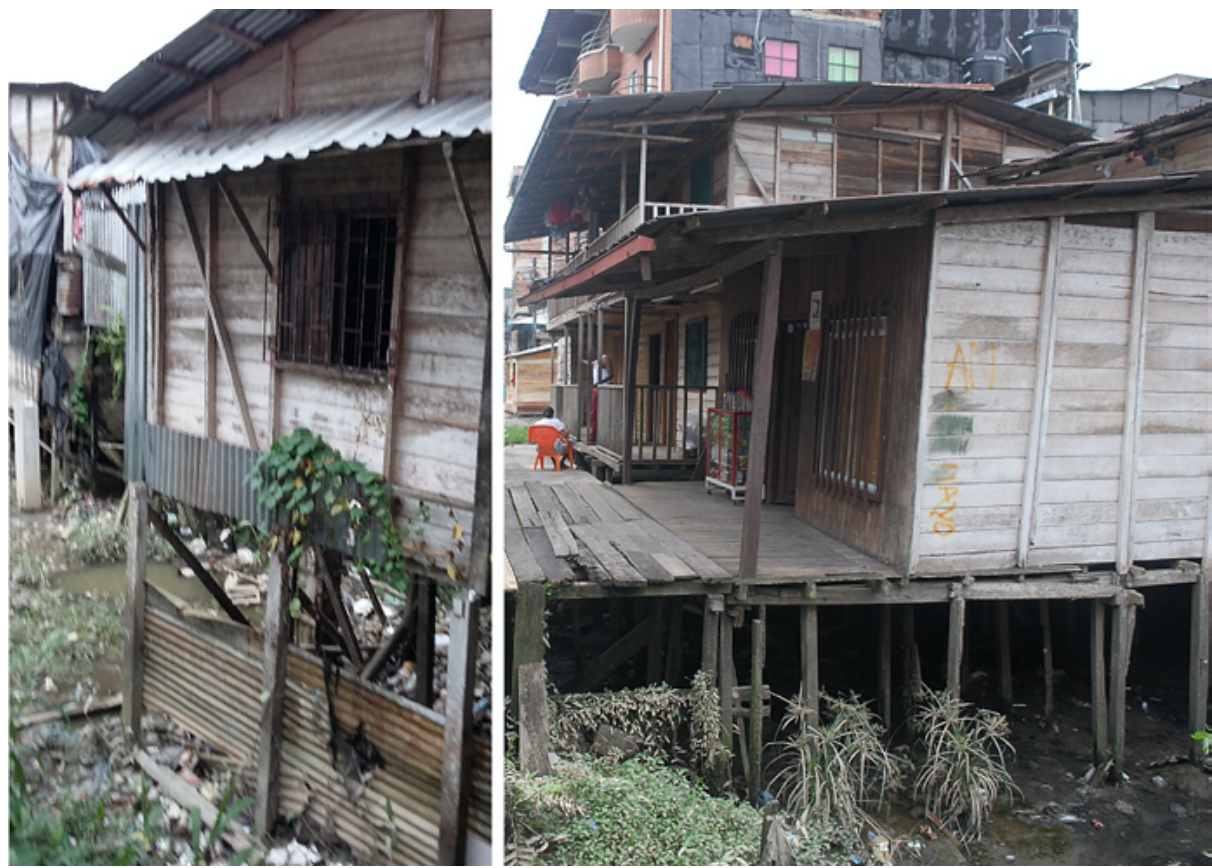

Fuente: elaboración propia. 
Además, la simplicidad y la nobleza de la arquitectura de madera siempre alienta un fuerte compromiso de aprendizaje, en particular, en los cursos de tecnología. Su evolución posterior como casas palafíticas (figura 9) invita a una reflexión valiosa sobre los procesos vernáculos que han estado dando forma a su ciudad y su adaptación al cambio climático.

\section{Reflexión: trabajo de campo como laboratorio de aprendizaje incluyente}

La reflexión sobre el taller en Quibdó como un espacio para el aprendizaje y su estructura, puede caracterizarse como un modelo lineal de transmisión de conocimiento, e.g., la conferencia inicial enmarcó el trabajo de campo, en lugar de dejar que la experiencia del sitio construyera el proceso de enseñanza. Sin embargo, si el proceso de enseñanza y el tema a enseñar están bien sincronizados (Ingold, 2017), los talleres basados en el trabajo de campo pueden verse como un tipo de aprendizaje con y de quienes participaron, interrumpiendo los modelos lineales de transmisión del conocimiento.

La asimilación de información eventualmente se puede convertir en algo contextual y tener cierta incertidumbre, $y$ no se trata exclusivamente de información traída de otro lugar (metodología importada del posgrado de ESALA, Universidad de Edimburgo). Esto se debe al reconocimiento de que el aprendizaje no suele tomar una forma estandarizada, sino que son las "lecturas o interpretaciones erróneas" las que cambian creativamente las ideas, teorías y prácticas cuando se trasplantan a diferentes contextos (Said, 1993; Jazeel \& McFarlane, 2010). La discusión con los estudiantes en Quibdó reveló posibles caminos de aprendizaje en este sentido, que podrían enriquecerse aún más con cursos de conservación o proyectos de diseño relacionados con la arquitectura histórica.

Por otro lado, en los cursos formales de posgrado hay más espacio dedicado para la alineación de la teoría y la práctica, como también para el aprendizaje de los temas desde diferentes perspectivas. Los estudiantes de la asignatura “Tecnología de la conservación” de la Universidad de Edimburgo, encuentran muy pertinente la discusión abierta sobre intervenciones de diseño y las opciones a sus propuestas, que ocurre al final de la serie de conferencias. El curso considera que es el momento adecuado, una vez los estudiantes se vuelven conocedores, suficientemente sensibles a los problemas y sus opiniones han madurado. También es cierto que los estudiantes del curso parecen a menudo ser conservadores en su enfoque durante el trabajo del curso, al mostrar creatividad y curiosidad limitadas. Si bien esto hace que los estudiantes sigan los requerimientos del curso muy de cerca, introducir a priori una mirada crítica a través de situaciones desconocidas que alteran las rutas de aprendizaje lineales, tersitorios 44 Especial 147 


\section{territorias} 44 Especial 148 pueden conducir a una mayor apropiación del conocimiento.

Los aspectos clave en los que debe centrarse la pedagogía en conservación son la apertura a la realidad, la incertidumbre de la información que produce un sitio y la capacidad de asignarles prioridades significativas (ICOMOS, 1993). Lo anterior, debido a la variedad de disciplinas de los estudiantes de posgrado, los procesos transformadores que promueven los currículos de Geografía o Arquitectura de pregrado, que en general, no pueden aplicarse allí. Aunque desde el punto de vista de la inclusión, los ejercicios cortos e intensivos de trabajo de campo pueden ofrecer sus propios métodos pedagógicos y generar un aprendizaje experimental dirigido por el alumno que puede abrir una investigación crítica, permitiendo que las condiciones de un sitio determinen la dirección del conocimiento, incluso en áreas muy enfocadas del campo. Por lo tanto, se pueden introducir más activamente como módulos dentro de asignaturas más grandes. No obstante, el campo de la conservación aún requiere ciertas habilidades técnicas y profesionales para respaldar estudios confiables y decisiones de intervención sostenibles. El trabajo de campo demanda recursos, pero promueve una conciencia crítica del espacio construido y automotivación que puede hacer que la enseñanza de estas habilidades sea mucho más rentable en un contexto más amplio. Completar un ejercicio que no es técnicamente exigente puede hacer que los estudiantes se sientan seguros y curiosos de explorar el contenido teórico o técnico con mucha más propiedad.

De forma alternativa, los estudiantes de pregrado en Arquitectura pueden ser inducidos de manera más efectiva a la conservación a través del trabajo de campo, con un mínimo de introducción al contexto teórico y técnico que les permita que el campo se convierta en otra área del portafolio del pensamiento crítico desarrollado a través de la exploración de sus sentidos.

\section{Conclusiones}

El trabajo de campo guiado por docentes de ambas universidades (Edimburgo-UTCh) a estudiantes de pregrado en Colombia tuvo como resultado:

- La estimulación de la autonomía en el pensamiento crítico de los alumnos y el desarrollo de nuevas habilidades.

- El incremento en la comprensión del patrimonio de madera.

- Razonamientos analíticos en sus proyectos de diseño, enriquecidos por una conciencia renovada del contexto urbano e interés de usar el centro tradicional como laboratorio para sus estudios.

Además, la reflexión posterior de la experiencia del taller mostró áreas más amplias donde el aprendizaje a través del trabajo de campo en conservación 
arquitectónica puede mejorarse. Más allá de enfocarse en las habilidades técnicas o la recopilación de datos como generalmente se hace en la actualidad, se puede abordar como una actividad intensiva para: (1) introducir de una manera emotiva a los estudiantes a la disciplina o (2) una oportunidad para desarrollar estrategias que aumentan la investigación crítica y el sentido de pertenencia en el aprendizaje. Esta metodología de aprendizaje puede potenciar la participation inclusiva en zonas donde las habilidades técnicas pueden escasear, mediante la estimulación de la curiosidad y la vivencia experiencial de los sentidos (e.g., observación).

El trabajo de campo en educación para la conservación arquitectónica puede ser alimentado sin dudas en cursos de pregrado por las actividades más desarrolladas y amplias en los currículos de Arquitectura y Geografía, donde se enseña cómo “observar lo existente" o estudiar sus agentes y culturas, y vincularlos a patrones en el entorno natural. Tales actividades pueden mejorar la apropiación del conocimiento de los estudiantes de conservación y su desarrollo como investigadores autónomos (QAA Scotland, 2012).

Asimismo, el trabajo de campo intensivo puede modificar los patrones lineales de aprendizaje en conservación arquitectónica para permitir que los estudiantes se involucren más estrechamente con los datos y las condiciones de un sitio, enriqueciendo su experiencia de aprendizaje, pero también preparándolos mejor para manejar lo inesperado en los proyectos de su vida profesional. Además, puede promover el desarrollo personal y la experiencia de la esencia de un lugar combinando la recopilación de datos primarios con formas menos estructuradas para crear narrativas personales de sus condiciones.

Esta ruptura de patrones lineales de aprendizaje también estimula la creatividad a través de la generación de espacios para la transformación de la práctica personal, de acuerdo con el trabajo del curso, el campo estudiado y el desafío a la teoría. Incluso, podría llevar a la creación de sus propios instrumentos o métodos de simulación para comprender un tipo de patología o una respuesta ambiental, ya que se reduce la mediación (o interferencia) en las impresiones del estudiante acerca de las condiciones y recursos que han creado un ambiente. Esto, en un territorio marginalizado como Quibdó, podría formar actitudes propias, innovadoras y sostenibles dependiendo de la duración e intensidad del trabajo de campo y el apoyo de lecturas teóricas de orientación.

Los cursos de pregrado en arquitectura son lo suficientemente largos como para desarrollar habilidades críticas de investigación y tecnología y, los estudiantes pueden introducirse a la conservación a través de un módulo o unidad de diseño. Siendo un campo especialista en cursos como los de Edimburgo o el Reino Unido en general, sería necesaria una articulación con los criterios profesionales que los condicionan en cada contexto (Quibdó, territarios 44 Especial 149 
Edimburgo). De otro lado, los cursos de posgrado de un año deben crear rápidamente un entorno de aprendizaje y experiencia, y la introducción temprana del trabajo de campo intensivo puede orientar firmemente a los estudiantes hacia la práctica multidisciplinaria de manera integral. Esta dimensión se explora últimamente en la asignatura de "Tecnología de restauración” en Edimburgo, con resultados prometedores. Como observó Christophe Girot, una experiencia de aprendizaje en el trabajo de campo debería, en última instancia, alimentar el impacto de ese momento singular del primer encuentro con un sitio.

\section{Glosario}

- Transmisión lineal de conocimiento: modelo que asume que los estudiantes aprenden directamente después de una clase y tutorías, siguiendo objetivos muy bien definidos.

- Levantamiento arquitectónico: técnicas para obtener la geometría y dimensión de edificios existentes.

- Evaluación del patrimonio cultural: identificación de los valores de interés patrimonial de bienes arquitectónicos.

- Viviendas palafíticas: casas de madera construidas sobre una serie de pilares, generalmente sobre cuerpos de agua o zonas inundables.

territarias 44 Especial 150 representados en los programas académicos de países o zonas que han sido previamente colonizadas.

\section{Agradecimientos}

Los autores reconocen amablemente el apoyo financiero para la investigación del Newton Caldas Fund del Consejo Británico.

Además, el primer autor tuvo el apoyo generoso del PEAK Urban programme, financiado por el UKRI's Global Challenge Research Fund, Grant Ref: Es/ P011055/1.

De la misma manera, se agradece calurosamente a los colegas de la UTCh: Johana Lozano, por coordinar el ejercicio; Edison Ledesma, director de la escuela por su hospitalidad; los tutores de los 3 grupos, arquitectos Aura Conto, José Luis Copete y Antonio Hinestroza por guiar a los estudiantes; Víctor Valencia Abadía, fundador de la escuela, por su entusiasta aportación; Douglas Cujar, por su inmenso conocimiento práctico de la evolución de la ciudad. También a la Diócesis del Chocó, por la inspiradora visita al sitio de la Catedral de Quibdó.

Al Dr. Dan Swanton, investigador principal del proyecto, quien proporcionó una visión única de los procesos de aprendizaje no lineal. A la profesora Suzanne Ewing, a la Dra. Lisa Moffit, a la Dra. Ana Bonet-Miro, al Dr. Miguel Paredes-Maldonado, a la Dra. Ruxandra-Iulia Stoica y a Jane Robertson, colegas de ESALA, 
y a la Profesora Donatella Fiorani, de la Universidad de Sapienza, se les agradece por su retroalimentación basada en sus inspiradoras prácticas docentes.

\section{Referencias}

Arango, S. (1993). La recuperación del pasado. En Historia de la Arquitectura en Colombia (pp. 256-262). Universidad Nacional de Colombia.

Bland, K., Chambers, B., Donert, K., \& Thomas, T. (1996). Fieldwork. En P. Bailey \& P. Fox (Eds.), Geography Teachers' Handbook (pp. 165-175). Geographical Association.

Bonet, A. (2019). Architectural design: ANY place. Assignment 3. ESALA, University of Edinburgh.

Brandi, C. (2005). Theory of restoration [Teoria del restauro, 1965]. Nardini Editore.

Carbonara, G. (2011). Architettura d'oggi e restauro: un confronto antico-nuovo. UTET scienze tecniche.

Carbonara, G. (2012). An Italian contribution to architectural restoration. Frontiers of Architectural Research, 1(1), 2-9. https://doi.org/10.1016/j. foar.2012.02.007

Cujar, D. (2015). Historia de Quibdó: 100 años de desarrollo urbano 1900-2000. Museo de Historia Natural de la Escuela Normal de Varones de Quibdó. http://es.slideshare.net/udelchoco/ exposicin-historia-de-quibdo-100-aosde-desarrollo-urbano-19002000
Dunphy, A., \& Spellman, G. (2009). Geography fieldwork, fieldwork value and learning styles. International Research in Geographical and Environmental Education, 18(1), 19-28. https://doi. org/10.1080/10382040802591522

Ewing, S. (2012). Architecture in context. Habits of seeing, knowing, and working with the existing in architecture. NORDIC Journal of Architecture, 2(3), 17-25.

Fiorani, D. (2019). Architectural Restoration Between Continuity and Innovation. The Italian Proposal. En C. di Biase (Ed.), European Schools in the teaching of Restoration. Tradition, perspectives (pp. 161-174). Politecnico di Milano.

Foskett, N. (1997). Teaching and learning through fieldwork. En D. Tilbury \& M. Williams (Eds.), Teaching and Learning Geography (pp. 189-201). Routledge.

Foskett, N. (1999). Forum: fieldwork in the geography curriculum-international perspectives and research issues. International Research in Geographical and Environmental Education, 8(2), 159-163. https://doi. org/10.1080/10382049908667603

France, D., \& Haigh, M. (2018). Fieldwork@40: fieldwork in geography higher education. Journal of Geography in Higher Education, 42(4), 498-514. https://doi.org/10.1080/03098265 .2018 .1515187 territarios 44 Especial 
Fuller, I., Edmonson, S., France, D., Higgit, D., \& Ratinen, I. (2006). International Perspectives on the Effectiveness of Geography Fieldwork for Learning. Journal of Geography in Higher Education, 30(1), 89-101. https://doi. org/10.1080/03098260500499667

Gold, J.R. (1991). Fieldwork. En JR. Gold,

A. Jenkins, R. Lee, J. Monk, J. Riley, I. Sheppard \& D. Unwin (Eds.), Teaching Geography in Higher Education: a manual of good practice (pp. 21-35). Blackwell.

González, L. (2003). Quibdó: contexto histórico, desarrollo urbano y patrimonio arquitectónico. Universidad Nacional de Colombia.

González, L. (2004). Evolución histórica de la arquitectura en madera en el Chocó. En Expresión formal de la vivienda espontánea. Serie Ciudad y Hábitat (11) Barrio Taller (pp. 17-40). Universidad Nacional de Colombia. https://repositorio.unal.edu.co/handle/unal/8412

Hope, M. (2009). The Importance of Direct Experience: A Philosophical Defence of Fieldwork in Human Geography. Journal of Geography in Higher Education, 33(2), 169-182. https://doi. org/10.1080/03098260802276698

Huerta, S. (s.f.). Fuentes para la Historia de la Construcción. Escuela Técnica Superior de Arquitectura de Madrid. http://biblioteca.aq.upm.es/fondoantiguo/historiaconstruccion.html
ICOMOS. (1993). Guidelines for Education and training in the conservation of Monuments. Ensembles and Sites.

Ingold, T. (2017). Anthropology and/as Education. Routledge.

Jazeel, T., \& McFarlane, C. (2010). The limits of responsibility: a postcolonial politics of academic knowledge production. Transactions of the Institute of British Geographers, 35(1), 109-124. https://doi.org/10.1111/j.14755661.2009.00367.x

Jokilehto, J. (2007). An International Perspective to Conservation Education. Built environment, 33(3), 275-286. https://doi.org/10.2148/ benv.33.3.275

Kent, M., Gilbertson, D., \& Hint, C. (1997). Fieldwork in Geography Teaching: a critical review of the literature and approaches. Journal of geography in Higher Education, 21(3), 313-332. https://doi. org/10.1080/03098269708725439

Lasheras, F. (1998). Patología de la madera. En Tratado de rehabilitación (vol. 3). Patología y técnicas de intervención. Elementos estructurales (pp. 255-276). ETSA Madrid; Editorial Munilla-Leria. Le Heron, R., Baker, R., \& McEwen, L. (2006). Co-learning: Re-linking Research and Teaching in Geography. Journal of Geography in Higher Education, 30(1), 77-87. https://doi. org/10.1080/03098260500499659

Edwar Calderón, Dimitris Theodossopoulos 
Lozano, J. (s.f.). Tipología de viviendas en zonas inundables y no inundables en la ciudad de Quibdó. Universidad Tecnológica del Chocó.

Matero, F. (2007). Loss, compensation, and authenticity: the contribution of Cesare Brandi to architectural conservation in America. Future Anterior, 4(1), 45-58. https://repository.upenn.edu/cgi/viewcontent. cgi ?article $=1010 \&$ context $=$ hp_papers

Moffit, L. (2019). The streamlines, vortices and plumes of the Blue Lagoon and Bath. Year 1, Master of Architecture. ESALA, University of Edinburgh.

Musso, S., \& De Marco, L. (Eds.). (2007). Teaching conservation/restoration of the architectural heritage. EAAE transactions on architectural education N. 38 .

Nairn, K. (2005). The problems of utilizing 'direct experience' in geography education. Journal of Geography in Higher Education, 29(2), 293-309. https://doi. org/10.1080/03098260500130635

Paredes-Maldonado, M., \& Bonet-Miro, A. (2018). Unit 3: Data-Driven Urban Prototypes, Architectural. Design 3: Explorations, MA(Hons) Architecture. ESALA, University of Edinburgh.

Pawson, E., Fournier, E., Haigh, M., Muniz, O., Trafford, J., \& Vajoczki, S. (2006). Problem-based learning in geography: Towards a critical assessment of its purposes, benefits and risks. Journal of Geography in Higher
Education, 30(1), 103-116. https://doi. org/10.1080/03098260500499709 QAA Scotland. (2012). What is mastersness? Report of the Scottish Higher Education Enhancement Committee Learning from International Practice. The Postgraduate Taught Student Experience Working Group. SHEEC.

Said, E. (1993). Culture and imperialism. Chatto and Windus.

Scott I., Fuller I., \& Gaskin S. (2006). Life without Fieldwork: Some Lecturers' Perceptions of Geography and Environmental Science Fieldwork. Journal of Geography in Higher Education, 30(1), 161-171. https://doi. org/10.1080/03098260500499832

Smith, P. (1987). Outdoor education and its educational objectives. Geography, 72(2), 209-216. https://www.jstor. org/stable/40571620

Universita di Roma "La Sapienza" (2019). Scuola di Specializzazione in Beni Architettonici e del Paesaggio. https://web.uniromal.it/ specialrestauro/percorso-restauro-deibeni-architettonici-e-del-paesaggio/ percorso-restauro-dei-beni-architettonici

University of Rome Tor Vergata. (2010). Summer School in Conservation of Historical, Monumental and Archaeological Sites. Organised by prof. Donato Abruzzese. https://www.facebook. com/ISSCoHMAS/

University of Edinburgh. (2020). Urban Conservation: Degree regulations and tersitarios 44 Especial 
programmes of study (DRPS). http:// www.drps.ed.ac.uk/20-21/dpt/ cxarchl1196.htm

University of Edinburgh. (2020). Conservation Technology. Degree regulations and programmes of study (DRPS). University of Edinburgh. http://www.drps.ed.ac. uk/20-21/dpt/cxareal 1017.htm

Universidad Tecnológica del Chocó. (2017, 27 de abril). Taller Internacional para
Aprender a Conservar el Patrimonio de Quibdó. https://www.utch.edu.co/ portal/es/noticias/1633-taller-internacional-para-aprender-a-conservar-elpatrimonio-de-quibd $\% \mathrm{C} 3 \% \mathrm{~B} 3 . \mathrm{html}$

Venturi, R., Scott-Brown, D., \& Izenour, S. (1972). Learning from Las Vegas. MIT Press. 